\title{
Taking the next step in publication productivity analysis in pediatric neurosurgery
}

\author{
Ryan P. Lee, MD, ${ }^{1}$ Raymond Xu, BS, ${ }^{2}$ Pooja Dave, ${ }^{3}$ Sonia Ajmera, BS, ${ }^{2}$ Jock C. Lillard, BS, ${ }^{2}$ \\ David Wallace, BS, ${ }^{2}$ Austin Broussard, BS, ${ }^{2}$ Mustafa Motiwala, BS, ${ }^{2}$ Sebastian Norrdahl, MS, ${ }^{2}$ \\ Carissa Howie, ${ }^{3}$ Oluwatomi Akinduro, BS, ${ }^{2}$ Garrett T. Venable, MD, ${ }^{4}$ Nickalus R. Khan, MD, ${ }^{4}$ \\ Douglas R. Taylor, MD, ${ }^{4}$ Brandy N. Vaughn, RN, ${ }^{5}$ and Paul Klimo Jr., MD, MPH ${ }^{4-6}$
}

\begin{abstract}
'Department of Neurosurgery, Johns Hopkins University School of Medicine, Baltimore, Maryland; ${ }^{2}$ College of Medicine and ${ }^{4}$ Department of Neurosurgery, The University of Tennessee Health Science Center, Memphis; ${ }^{3}$ Rhodes College, Memphis; ${ }^{5} \mathrm{Le}$ Bonheur Children's Hospital, Memphis; and ${ }^{6}$ Semmes Murphey, Memphis, Tennessee
\end{abstract}

OBJECTIVE There has been an increasing interest in the quantitative analysis of publishing within the field of neurosurgery at the individual, group, and institutional levels. The authors present an updated analysis of accredited pediatric neurosurgery training programs.

METHODS All 28 Accreditation Council for Pediatric Neurosurgery Fellowship programs were contacted for the names of pediatric neurosurgeons who were present each year from 2011 through 2015. Faculty names were queried in Scopus for publications and citations during this time period. The 5-year institutional Hirsch index [ih(5)-index] and revised 5-year institutional $h$-index [ir(5)-index] were calculated to rank programs. Each publication was reviewed to determine authorship value, tier of research, clinical versus basic science research, subject matter, and whether it was pediatrics-specific. A unique 3 -tier article classification system was introduced to stratify clinical articles by quality and complexity, with tier 3 being the lowest tier of publication (e.g., case reports) and tier 1 being the highest (e.g., randomized controlled trials).

RESULTS Among 2060 unique publications, 1378 (67\%) were pediatrics-specific. The pediatrics-specific articles had a mean of 15.2 citations per publication (median 6), whereas the non-pediatrics-specific articles had a mean of 23.0 citations per publication (median $8 ; p<0.0001$ ). For the $46 \%$ of papers that had a pediatric neurosurgeon as first or last author, the mean number of citations per publication was 12.1 (median 5.0) compared with 22.5 (median 8.0) for those in which a pediatric neurosurgeon was a middle author $(p<0.0001)$. Seventy-nine percent of articles were clinical research and $21 \%$ were basic science or translational research; however, basic science and translational articles had a mean of 36.9 citations per publication (median 15) compared with 12.6 for clinical publications (median 5.0; $p<0.0001$ ). Among clinical articles, tier 1 papers had a mean of 15.0 citations per publication (median 8.0), tier 2 papers had a mean of 18.7 (median 8.0), and tier 3 papers had a mean of 7.8 (median 3.0). Neuro-oncology papers received the highest number of citations per publication (mean 25.7). The most common journal was the Journal of Neurosurgery: Pediatrics (20\%). MD/ $\mathrm{PhD}$ faculty members had significantly more citations per publication than MD faculty members (mean 26.7 vs 14.0; $p<$ 0.0001 ) and also a higher number of publications per author (mean 38.6 vs 20.8). The median ih(5)- and ir(5)-indices per program were 14 (range 5-48) and 10 (range 5.6-37.2), respectively. The mean ir(5)/ih(5)-index ratio was 0.8 . The top 5 fellowship programs (in descending order) as ranked by the ih(5)-index corrected for number of faculty members were The Hospital for Sick Children, Toronto; Children's Hospital of Pittsburgh; University of California, San Francisco Benioff Children's Hospital; Seattle Children's Hospital; and St. Louis Children's Hospital.

CONCLUSIONS About two-thirds of publications authored by pediatric neurosurgeons are pediatrics-specific, although non-pediatrics-specific articles averaged more citations. Most of the articles authored by pediatric neurosurgeons are clinical, with basic and translational articles averaging more citations. Neurosurgeons with PhD degrees averaged more total publications and more citations per publication. In all, this is the most advanced and informative analysis of publication productivity in pediatric neurosurgery to date.

https://thejns.org/doi/abs/10.3171/2018.1.PEDS17535

KEY WORDS bibliometrics; pediatric; neurosurgery; ih(5); ranking

ABBREVIATIONS ACPNF = Accreditation Council for Pediatric Neurosurgery Fellowship; $h$-index $=$ Hirsch index; i $h(5)$-index $=5$-year institutional Hirsch index; ir(5)-index $=$ revised 5 -year institutional $h$-index; $r$-index $=$ revised $h$-index.

SUBMITTED October 2, 2017. ACCEPTED January 2, 2018.

INCLUDE WHEN CITING Published online March 23, 2018; DOI: 10.3171/2018.1.PEDS17535. 
$\mathrm{R}$ ECENTLY, greater attention has been paid to quantifying and comparing publication productivity between individuals and institutions. With this increased focus on objectively measuring and comparing output, many have sought to provide more advanced statistics beyond simply reporting the number of publications and citations. This has resulted in the expansion and evolution of bibliometrics, the science of the statistical analysis of written publications. ${ }^{3,4,28}$ The most popular bibliometric standard is the Hirsch index ( $h$-index), which combines the productivity and citation impact of an individual into a single metric., ${ }^{1,6,8}$ Originally applied to individual authors, the $h$-index has also been applied extensively to institutions to compare them for the purposes of ranking and funding allocation. ${ }^{2,30}$ Numerous analyses have since computed individual and institutional $h$-indices for benchmark and comparison in the field of neurosurgery. ${ }^{7,-12,14,16,18,22,25,26,29,31-33}$

One limitation of the raw (or unadjusted) $h$-index is that it includes all articles that an author or group of authors has ever published. In 2014, we found the median $h$-index of 312 North American pediatric neurosurgeons to be $10 .{ }^{12}$ Because the unadjusted $h$-index is summed, it does not adequately reflect recent productivity and citation impact, or the lack thereof. To account for this in the setting of institutional comparisons, the 5 -year institutional $h$-index, or i $h(5)$-index, was created. ${ }^{27}$ This index captures the $h$-index of a whole group, department, or institution-as determined by the user-over a discrete contemporary 5-year period. It was recently validated and applied to neurosurgical departments with residency training programs in the United States and Canada but has not yet been applied to pediatric neurosurgery fellowship programs. ${ }^{17,18,27}$

Another limitation of the $h$-index is that it does not take into account the degree of contribution of the author to the publication. For neurosurgical publications, the first, second, and last authors typically contribute disproportionately more to the project than the middle author(s). This contribution may not only be in terms of time, but also in resources (e.g., laboratory space, equipment, funding) and project oversight. On the other hand, "gift" middle authorship is a well-established practice in neurosurgery. ${ }^{13}$ The revised $h$-index ( $r$-index), was recently introduced to provide more weight to first or last authorship as compared with middle authorship. ${ }^{23}$ More specifically, the $r$-index is simply an $h$-index with first or last authorship weighted 4 times as much as middle authorship.

In this study, we applied, for the first time, the i $h(5)$ index to the 28 Accreditation Council for Pediatric Neurosurgery Fellowship (ACPNF)-approved pediatric neurosurgery fellowship programs in North America to capture and compare contemporary publication productivity. Previously, we ranked the 26 ACPNF-approved programs that were in existence in 2014 by summing the number of publications and citations from those publications from 2008 through 2013 and found that the top 2 institutions were Harvard University (Boston Children's Hospital) and University of Toronto (The Hospital for Sick Children). ${ }^{12}$ In this report, we also introduce the revised 5-year institutional $h$-index (ir(5)-index), which is analogous to the i $h(5)$-index but takes into account au- thorship value using the $r$-index formula and uses this metric to quantify and compare program output. Lastly, in an attempt to provide programs with guidance as to how to allocate resources and attention when seeking to improve citation impact, we analyzed article and faculty member variables that correlate to productivity.

\section{Methods}

\section{Selection of Programs and Collection of Faculty Information}

A list of the 28 currently accredited US and Canadian pediatric neurosurgery fellowship programs was obtained from the ACPNF website (www.acpnf.org). All programs were successfully contacted in May 2017 to obtain a list of pediatric neurosurgeons who practiced at their institutions on a year-by-year basis from 2011 through 2015. The academic rank and advanced degrees of the faculty members were also collected. The number of cases per program per year was also obtained from the ACPNF website or from direct contact with the program.

\section{Collection of Publication and Citation Data}

Faculty names were queried in Scopus using a methodology similar to that used in our previous publications,,${ }^{17,27}$ with some notable methodologic improvements. Results were filtered to include only those articles published during the time frame in which the faculty member was present at the particular institution in question. Both original articles and review articles were included, whereas review articles were excluded in our previous publications. ${ }^{12,17,27}$ Each publication result was then reviewed to confirm the correct affiliation for the author search. Great care was taken to capture every article for each faculty member. Finally, the number of citations per article was collected from the time of article publication through June 2017. This is a departure from our previous methodology and was modified to allow 18 months of additional time for citation accumulation, which is particularly important for those articles published in 2015-the last year of the publication time period.

An article with multiple pediatric neurosurgeon authors was attributed to an institution and the individuals as follows: 1) if 2 or more authors were from the same institution, the article was counted only once for that institution in the institutional comparison analysis; 2) if 2 or more authors were from 2 or more institutions, each institution received credit for that article but only once and only if the faculty member was affiliated with the institution during the year of publication; and 3) each faculty author of a publication received credit for that publication in the analysis of individual faculty members that considered rank, degree, and so on (Table 1).

\section{Defining Article Characteristics}

To analyze the article characteristics, all publications from each faculty member included in this analysis were pooled into a single database. Duplicates (e.g., 2 authors who contributed to the same article) were removed so that an article was not double counted, which would skew the statistics for a particular variable. 
TABLE 1. Top 10 most common journals

\begin{tabular}{|c|c|c|c|c|c|c|c|}
\hline \multirow[b]{2}{*}{ Characteristic } & \multirow{2}{*}{$\begin{array}{l}\text { Impact } \\
\text { Factor* }\end{array}$} & \multicolumn{2}{|c|}{ Citations per Publication } & \multicolumn{2}{|c|}{ Publications } & \multicolumn{2}{|c|}{ Citations } \\
\hline & & Mean* & Median* & No. & Percentage & No. & Percentage \\
\hline Journal of Neurosurgery: Pediatrics & 2.17 & 8.8 & 6 & 421 & 20 & 3713 & 10 \\
\hline Child's Nervous System & 1.081 & 6.0 & 3 & 162 & 8 & 972 & 3 \\
\hline Neurosurgery & 4.889 & 13.0 & 10 & 85 & 4 & 1101 & 3 \\
\hline World Neurosurgery & 2.592 & 5.8 & 3 & 56 & 3 & 326 & 1 \\
\hline Neurosurgical Focus & 3.139 & 7.3 & 3.5 & 50 & 2 & 366 & 1 \\
\hline Journal of Neuro-Oncology & 2.980 & 12.4 & 8 & 47 & 2 & 583 & 2 \\
\hline Journal of Neurosurgery & 4.059 & 15.1 & 9 & 41 & 2 & 618 & 2 \\
\hline Pediatric Neurosurgery & 0.550 & 2.9 & 2 & 36 & 2 & 105 & $<1$ \\
\hline Pediatric Blood \& Cancer & 2.513 & 11.5 & 8 & 33 & 2 & 379 & 1 \\
\hline Neuro-Oncology & 7.786 & 28.7 & 20 & 31 & 2 & 891 & 2 \\
\hline Top 10 journals & & 9.4 & 6.0 & 962 & 47 & 9054 & 25 \\
\hline All journals $(n=407)$ & & 17.8 & 7.0 & 2060 & 100 & 36,584 & 100 \\
\hline
\end{tabular}

For each article, we determined the following characteristics: whether a pediatric neurosurgeon was first or last author (compared with middle authorship status), the article tier (Table 2), the article subject, whether it was a pediatricneurosurgery-specific publication, where it was published and the 2017 impact factor of that journal, and whether it was a clinical, basic science, or translational study. The impact factor was obtained from the journal websites and cross-referenced with www.scijournal.org. As shown in Table 2, clinical articles were subdivided into 3 tiers based on the quality or complexity of the research in question. Tier 1 mostly consisted of controlled, high-quality, primary studies, tier 2 consisted of either secondary research (i.e., the study used previously collected or published data) or lower quality primary studies, and tier 3 consisted of the lowest quality and least complex publications including case reports, small series, survey-based studies, and technical reports. Articles that did not easily fit into one of these tiers were categorized by consensus. Basic and translational science articles were not subdivided by tier.

To determine if an article was pediatric-neurosurgeryspecific, the following criteria were used. For clinical articles, the publication methodology was reviewed to determine the age range of the study participants. If the age range included only pediatric patients (age range $0-21$ years), then it was considered pediatrics-specific. For basic and translational science papers, this distinction was more difficult. Many disease processes are inherently pediatricsspecific, such as craniosynostosis and myelomeningocele, and were thus classified as pediatrics-specific on that basis (unless specifically stated otherwise). If the topic was a disease process that occurs predominantly in children (e.g., medulloblastoma), then it was generally included as pediatrics-specific. Most articles classified as pediatricsspecific, however, were clearly designated as such in the title or paper methodology, similar to clinical articles. Two different authors (R.P.L. and the author assigned to primary data collection for that institution) reviewed each article and the senior author (P.K.) settled discrepancies.
To classify articles as clinical, basic science, or translational research, we used previously published definitions. ${ }^{24}$ In brief, clinical articles were patient oriented and included the following characteristics: "(a) mechanism of disease, (b) therapeutic interventions, (c) clinical trials, or (d) development of new technologies." Clinical articles also included the use of human tissues and specimens "for which an investigator (or colleague) directly interact[ed] with human subjects." Basic science research, on the other hand, centers on the "acquisition of knowledge without obligation to apply it to practical ends." Translational research lies in between and serves to bridge the gap by applying basic science discoveries to the clinical realm. Giv-

\section{TABLE 2. Clinical article tiers}

\begin{tabular}{l}
\hline Tier 1 \\
\hline Prospective \& retrospective controlled cohort \\
\hline Case-control study \\
\hline Randomized controlled trial \\
\hline Multicenter non-industry-sponsored study \\
\hline Tier 2 \\
\hline Secondary research \\
\hline Systematic review w/ meta-analysis \\
\hline Big database or registry based \\
\hline Uncontrolled case series (>10 patients) \\
\hline Multicenter but industry sponsored \\
\hline Observational \\
\hline Guidelines \\
\hline Tier 3 \\
\hline Case reports \\
\hline Review article \\
\hline Uncontrolled case series ( $\leq 10$ patients) \\
\hline Other (e.g., surgical simulation, cadaver studies, technology or \\
instrument design, survey) \\
\hline
\end{tabular}


TABLE 3. Citation counts by article subject

\begin{tabular}{|c|c|c|c|c|c|c|c|}
\hline \multirow[b]{2}{*}{ Characteristic } & \multicolumn{2}{|c|}{ Citations per Publication* } & \multirow[b]{2}{*}{ p Value } & \multicolumn{2}{|r|}{ Publications } & \multicolumn{2}{|r|}{ Citations } \\
\hline & Mean & Median & & No. & Percentage of Total $\dagger$ & No. & Percentage of Total $\dagger$ \\
\hline Tumor & 25.7 & 10.0 & $<0.0001$ & 731 & 35 & 18,782 & 51 \\
\hline Clinical & 12.1 & 6.0 & & 422 & $20(58)$ & 5108 & $14(27)$ \\
\hline Basic science or translational & 44.3 & 18.0 & & 309 & $15(42)$ & 13,674 & $37(73)$ \\
\hline Spine & 10.6 & 5.0 & 0.67 & 220 & 11 & 2337 & 6 \\
\hline Clinical & 10.7 & 5.0 & & 216 & $10(98)$ & 2305 & $6(99)$ \\
\hline Basic science or translational & 8.0 & 8.0 & & 4 & $<1(2)$ & 32 & $<1(1)$ \\
\hline Hydrocephalus & 8.7 & 5.0 & 0.13 & 191 & 9 & 1661 & 5 \\
\hline Clinical & 9.0 & 5.0 & & 177 & $9(93)$ & 1597 & $4(96)$ \\
\hline Basic science or translational & 4.9 & 2.5 & & 13 & $1(7)$ & 64 & $<1(4)$ \\
\hline Functional/epilepsy & 15.5 & 6.0 & 0.04 & 237 & 12 & 3684 & 10 \\
\hline Clinical & 14.9 & 6.0 & & 194 & $9(82)$ & 2895 & $8(79)$ \\
\hline Basic science or translational & 18.8 & 11.0 & & 41 & $2(18)$ & 789 & $2(21)$ \\
\hline Vascular & 11.7 & 6.0 & 0.006 & 169 & 8 & 1981 & 5 \\
\hline Clinical & 11.4 & 5.0 & & 159 & $8(94)$ & 1806 & $5(91)$ \\
\hline Basic science or translational & 17.5 & 15.0 & & 10 & $<1(6)$ & 175 & $<1(9)$ \\
\hline Trauma & 12.4 & 8.0 & 0.85 & 174 & 8 & 2156 & 0.06 \\
\hline Clinical & 12.5 & 8.0 & & 153 & $7(88)$ & 1915 & $0.05(89)$ \\
\hline Basic science or translational & 11.5 & 10.0 & & 21 & $1(12)$ & 241 & $0.01(11)$ \\
\hline Chiari malformation & 9.2 & 4.0 & 0.30 & 65 & 3 & 595 & 2 \\
\hline Clinical & 9.2 & 4.5 & & 64 & $3(98)$ & 590 & $2(99)$ \\
\hline Basic science or translational & 2.0 & 3.0 & & 2 & $<1(2)$ & 4 & $<1(1)$ \\
\hline Craniosynostosis & 9.5 & 4.0 & 0.88 & 77 & 4 & 735 & 2 \\
\hline Clinical & 9.7 & 4.0 & & 75 & $4(97)$ & 725 & $2(99)$ \\
\hline Basic science or translational & 5.0 & 5.0 & & 2 & $<1(3)$ & 10 & $<1(1)$ \\
\hline Total & 17.8 & 7.0 & & 2060 & 100 & 36,584 & 100 \\
\hline
\end{tabular}

* The $p$ value represents the comparison of the mean citations per publication between clinical versus basic or translational science publications within subjects.

$\dagger$ Number in parentheses represents the percentage of that particular subject, as opposed to the percentage of the total publications or citations.

en the difficulty in separating basic science research and translational research articles in many cases, we grouped them together in this analysis.

Articles were also classified into 1 of 8 subjects, if applicable: tumor, spine, hydrocephalus, functional/epilepsy, vascular, trauma, Chiari malformation, and craniosynostosis. An article could fall under multiple subjects, or none at all. Further subclassification was used to compare clinical articles with basic science and translational articles within each subject (Table 3).

\section{Calculation of Metrics and Ranking of Programs}

The first metric calculated and used for interdepartmental comparison was the i $h(5)$-index. ${ }^{6}$ It was calculated as follows: $\mathrm{i} h(5)=$ the $h$ number of publications with $\geq h$ number of citations. The pool of publications used for this metric included all those published by all authors from the institution in question during the 5-year period between January 1, 2011, and December 31, 2015. The citations for each publication were calculated cumulatively from January 1, 2011, through June 30, 2017.

This metric has been previously used to analyze the publication output from all US and Canadian neurosurgi- cal programs. ${ }^{17,27}$ The $\mathrm{i} h(5)$-index corrected for the number of faculty members was also calculated for each institution. We also corrected the i $h(5)$-index for authorship value using the $r$-index formula, which yielded the novel ir(5)index. The $r$-index formula values the first and last authorship positions at 4 times more than middle authorship: $r=$ $(1.2 \times a)+(0.4 \times h)$, where $a$ equals the subset of articles $h$ in which an author from the institution or program was first or last author. Programs were ranked by these 4 bibliometric measures: 1) i $h(5)$-index; 2) i $h(5)$-index/(number of faculty members); 3) ir(5)-index; and 4) ir(5)-index/ (number of faculty members). The primary ranking used was $\mathrm{i} h(5)$-index/(number of faculty members).

\section{Statistical Analysis}

All statistical analyses were carried out using JMP Pro (version 13, SAS Institute, Inc.). The mean and median numbers of citations per publication were calculated for each category or characteristic. The mean number of citations per publication was compared between characteristics using the Wilcoxon rank-sum test. The i $h(5)$-index and $i r(5)$-index were compared with linear regression. The impact factors of the top 10 journals were also compared 
TABLE 4. Overview of article characteristics

\begin{tabular}{|c|c|c|c|c|c|c|c|}
\hline \multirow[b]{2}{*}{ Characteristic } & \multicolumn{2}{|c|}{ Citations per Publication } & \multirow[b]{2}{*}{ p Value } & \multicolumn{2}{|r|}{ Publications } & \multicolumn{2}{|r|}{ Citations } \\
\hline & Mean & Median & & No. & Percentage of Total & No. & Percentage of Total \\
\hline Pediatrics-specific & & & $<0.001$ & & & & \\
\hline Yes & 15.2 & 6.0 & & 1378 & 67 & 20,892 & 57 \\
\hline No & 23.0 & 8.0 & & 682 & 33 & 15,692 & 43 \\
\hline Authorship & & & $<0.0001$ & & & & \\
\hline First or last & 12.1 & 5.0 & & 940 & 46 & 11,368 & 31 \\
\hline Middle & 22.5 & 8.0 & & 1120 & 54 & 25,216 & 69 \\
\hline Article Type & & & $<0.0001$ & & & & \\
\hline Clinical & 12.6 & 5.0 & & 1619 & 79 & 20,325 & 56 \\
\hline Basic science or translational & 36.9 & 15.0 & & 441 & 21 & 16,259 & 44 \\
\hline \multicolumn{8}{|l|}{ Clinical article tier } \\
\hline 1 & 15.0 & 8.0 & $0.47^{*}$ & 398 & $25 \S$ & 5974 & 29 \\
\hline 2 & 18.7 & 8.0 & $<0.0001 \dagger$ & 440 & $27 \S$ & 8234 & 41 \\
\hline 3 & 7.8 & 3.0 & $<0.0001 \ddagger$ & 781 & $48 \S$ & 6117 & 30 \\
\hline Total & 17.8 & 7.0 & & 2060 & 100 & 36,584 & 100 \\
\hline
\end{tabular}

with the mean and median numbers of citations per article for each journal using linear regression; $p \leq 0.05$ denoted significance for all statistical tests.

\section{Results}

\section{Article Characteristics}

Among the 28 programs, there were 2060 unique publications counted in this analysis during the 5-year period (January 1, 2011, to December 31,2015) with a total of 36,584 citations (from January 1, 2011, to June 30, 2017) (Table 4). The mean and median numbers of citations per publication were 17.8 and 7.0, respectively. There were 123 articles that had authors from multiple institutions counted in this comparison. Duplicates were excluded from the analysis of the article characteristics.

Of the 2060 total publications, 1378 (67\%) were pediatrics-specific. The mean and median numbers of citations per publication for pediatrics-specific articles were 15.2 and 6.0, respectively, compared with 23.0 and 8.0 for nonpediatrics-specific articles $(\mathrm{p}<0.0001)$.

Approximately $79 \%$ of articles were clinical, with mean and median numbers of citations per publication of 12.6 and 5.0, respectively. By contrast, the other $21 \%$ of articles, classified as basic or translational, had a mean of 36.9 and median of 15.0 ( $\mathrm{p}<0.0001)$. Forty-eight percent of the clinical articles were categorized as tier 3 , with a mean of 7.8 citations per publication (median 3.0). Tier 2 articles, which were $27 \%$ of the total, averaged 18.7 citations per publication (median 8.0). Tier 1 articles were $25 \%$ of total articles and averaged 15.0 citations per publication (median 8.0). Tier 1 and 2 articles each averaged significantly more citations than tier 3 articles $(p<0.0001)$ but neither averaged more than the other $(\mathrm{p}=0.47)$.
Pediatric neurosurgeons (affiliated with one of the 28 study institutions) were the first or last author for $46 \%$ of publications. The mean and median numbers of citations per publication for articles with a pediatric neurosurgeon as the first or last author were 12.1 and 5, respectively. For the other 54\% of publications, for which the pediatric neurosurgeon was a middle author, there were on average 22.5 citations per publication with a median of $8.0(\mathrm{p}<0.0001)$.

\section{Article Subject}

As shown on Table 3, we classified each article according to subject matter. "Tumor" was the most commonly published subject in this analysis and comprised approximately $35 \%$ of articles. The next most common subject was functional/epilepsy, which only made up $12 \%$ of articles. Tumor articles also had the highest number of citations per publication (mean 25.7; median 10), and they made up over half of all citations among all papers. Tumor articles also accounted for over $70 \%$ of all basic and translational science articles.

\section{Top Journals}

The top 10 journals from the analysis are shown in Table 1 . More than 400 journals were captured in this analysis, but almost half of the articles were published in just 10 journals. Notably, $20 \%$ of articles were published in just 1 journal, Journal of Neurosurgery: Pediatrics. The top 10 journals accounted for $47 \%$ of the total publications and $25 \%$ of the total citations and had a mean and median number of citations per publication of 9.4 and 6.0, respectively. Both the mean and median numbers of citations per publication for the top 10 journals were correlated with the journal impact factor on the linear regression analysis $\left(\mathrm{R}^{2}\right.$ mean 0.87, $\mathrm{p}<0.0001 ; \mathrm{R}^{2}$ median 0.85, $\mathrm{p}<0.001$ ). 


\section{Program Comparison and Ranking}

The median $\mathrm{i} h(5)$-index per program was 14.0 with a range of 5 to 48 (Table 5). The top 5 fellowship programs based on the $\mathrm{i} h(5)$-index corrected for number of faculty members (our primary ranking measure) were the following: 1) The Hospital for Sick Children; 2) Children's Hospital of Pittsburgh; 3) University of California, San Francisco Benioff Children's Hospital; 4) Seattle Children's Hospital; and 5) St. Louis Children's Hospital. Rankings were also calculated and are shown in Table 5 for uncorrected $\mathrm{i} h(5)$-index values.

We also created a novel bibliometric index, based on the $r$-index, which is a modified $h$-index and gives more weight to the first or last author of a publication. This new index, called the ir(5)-index, is the institutional $r$-index over the previous 5 years. Table 5 includes rankings based on the ir(5)-index and corrected ir(5)-index. The top 5 fellowship programs based on the ir(5)-index corrected for number of faculty members were the following: 1) The Hospital for Sick Children; 2) Children's Hospital of Pittsburgh; 3) St. Louis Children's Hospital; 4) Doernbecher Children's Hospital; and 5) University of California, San Francisco Benioff Children's Hospital. The ir(5)-index was correlated with the $i h(5)$-index on the linear regression analysis $\left(\mathrm{R}^{2}=0.88 ; \mathrm{p}<0.001\right)$.

Table 5 also includes a statistic that compares the value of the ir(5)-index with the i $h(5)$-index, the ir(5)-index/ ih(5)-index ratio. This statistic attempts to quantify the relative change in the program's index when first and last authorship are more heavily weighted. On average, the ratio was 0.8 , meaning that the ir(5)-index was $80 \%$ that of the i $h(5)$-index for the typical program. Doernbecher Children's Hospital had the highest ir(5)-index/ih(5)-index ratio at 1.24 , meaning the ir(5)-index was $124 \%$ of the i $h(5)$-index.

There was a notable variability among the metrics as seen in Table 5. However, The Hospital for Sick Children and Children's Hospital of Pittsburgh were consistently ranked first and second, respectively, in every metric. Comparing the uncorrected to the corrected i $h(5)$-index, the mean change in rank was 3.8 (range -14 to 8 ). For the uncorrected to corrected ir(5)-index, the mean change in rank was 3.5 (range -10 to 8 ). Between the corrected $i h(5)$ and corrected ir(5), the mean change in rank was 4 (range -14 to 12 ).

\section{Academic Rank and Degree}

The articles authored by $\mathrm{MD} / \mathrm{PhD}$ faculty members had a significantly higher mean number of citations per publication than those authored by MD-only (or DO-only) faculty members (26.7 vs 14.0; p < 0.0001) (Table 6). Each MD author on average produced 20.8 publications compared with 38.6 for each $\mathrm{MD} / \mathrm{PhD}$ author. As academic rank increased from assistant professor to associate professor to professor, the faculty member had significantly more citations per publication (8.5 vs 13.6 vs 21.7 , respectively; $\mathrm{p}<0.001)$. The mean number of publications was also greater at each level of more highly ranked faculty members (9.7 vs 26.0 vs 35.9, respectively). Interestingly, almost $40 \%$ of the pediatric neurosurgeons in these 28 programs were full professors.

\section{Discussion \\ Methodology}

Several major methodological changes were implemented in the data collection process for this paper compared with previous publications. ${ }^{12,15,27}$ First, citations were counted for 18 months after the discrete 5-year period ended-in this case through mid-2017-as opposed to ending citation collection concurrently with publication collection as in our previous reports. Madhugiri et al. demonstrated that the time for neurosurgical articles to peak in citations is about 6.25 years after publication. ${ }^{19}$ Therefore, later articles in the discrete publication collection time period do not have adequate time to accumulate citations. We felt that adding approximately 18 months was a fair compromise that allowed us to present a metric that encompassed a contemporary time frame and also allowed later articles in the discrete time frame to accumulate citations so as to more adequately reflect the impact or interest generated by that article. Therefore, early articles have approximately 5-6 years and later articles have 2-3 years of citation follow-up by using this new methodology. Moreover, it has also been demonstrated that the time to peak citation does not differ between articles in neurosurgical journals compared with those in nonneurosurgical high-impact journals, so there should be no bias in our methodology against either. ${ }^{19}$

The second methodological improvement was to directly contact every program to obtain faculty affiliations for each year in the discrete time frame. Previously, faculty names were collected from program websites. However, this led to a limitation in that, if a faculty member left an institution before the website was accessed at the end of the 5-year period, the individual's contributions may not have been credited to the institution he or she just left even if that individual had been there for all of the 5 years. To overcome this limitation, we asked programs to provide a year-by-year listing of which faculty members were present so that each institution with whom a faculty member was affiliated during the 5-year period would get credit for the publications published during those years.

Third, we took a critical step by factoring in authorship value. This was accomplished by incorporating the $r$-index, which is a modified version of the $h$-index that weighs first or last authorship 4 times more than middle authorship. The ir(5)-index is introduced as an analogous index to the $i h(5)$-index for this very purpose.

Last, we included review articles in the analysis, whereas previously they were excluded. These reports, while not primary research, can still require significant time and effort that should be acknowledged. They are appropriately classified in the lowest tier to account for the lack of primary research, unless they included a meta-analysis, which would upgrade them to tier 2.

\section{Article Characteristics}

There were over 300 more articles across this updated 5 -year time period compared with our previous articles for 2009-2013. ${ }^{12}$ This increase is due to a combination of globally increased academic productivity within pediatric neurosurgery and the addition of 2 new fellowship pro- 


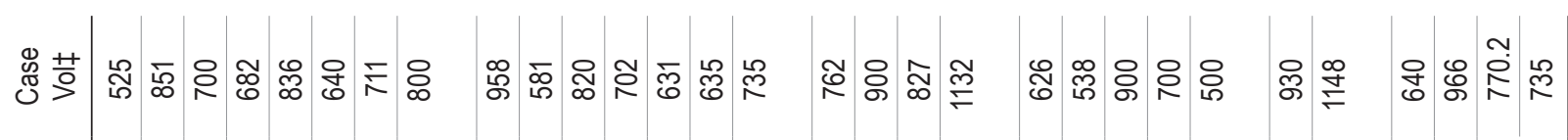

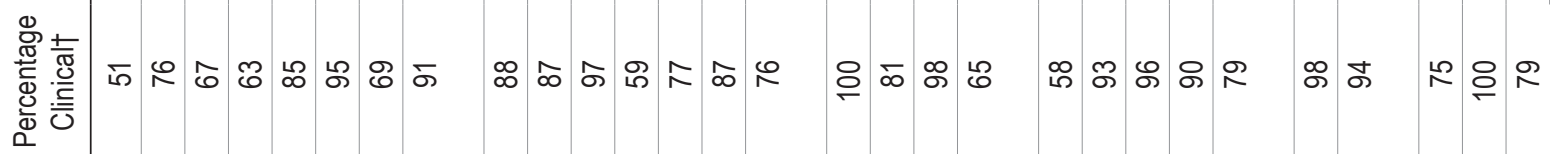

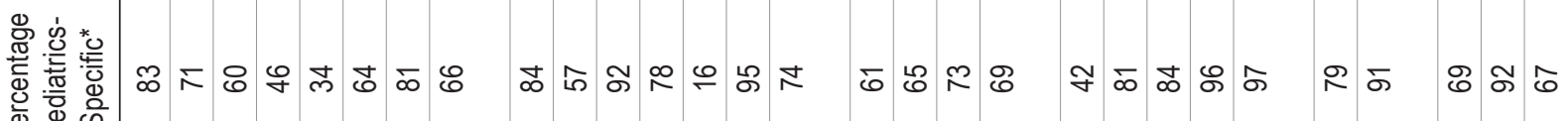
离离

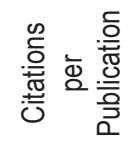

กำ ஸ் ஹீ

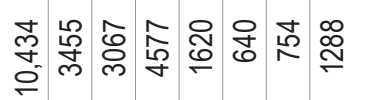

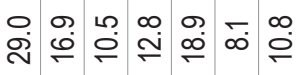

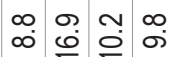

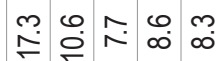

F尔

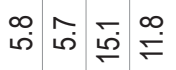
畩

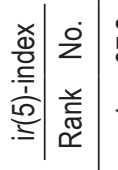

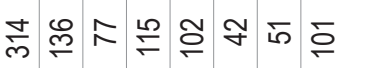

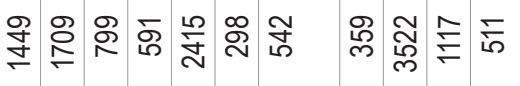

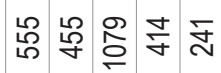

ஓㅇำ

웅 ก

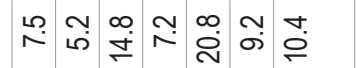
ㅈำกงกำ

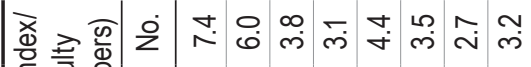

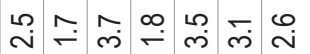
$\ulcorner$ ก⿻上ก는

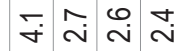
$\checkmark \simeq \stackrel{\infty}{\circ}$ 旁兽 立武交点希

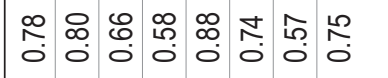

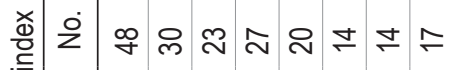
旁旁总

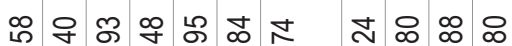
0,00000

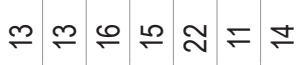

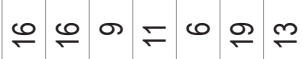

으윤ㄷㄴ $\approx \sim \mp$ พ

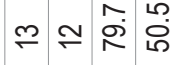

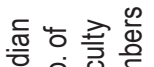

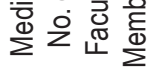

Gீ

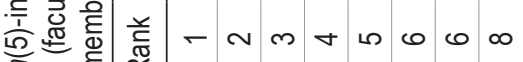

\section{$\infty$} $\infty \infty \leftleftarrows \cong \stackrel{m}{m}$

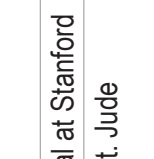

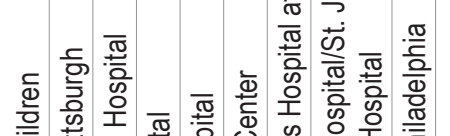

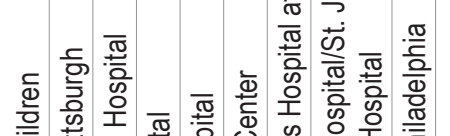

휸 要

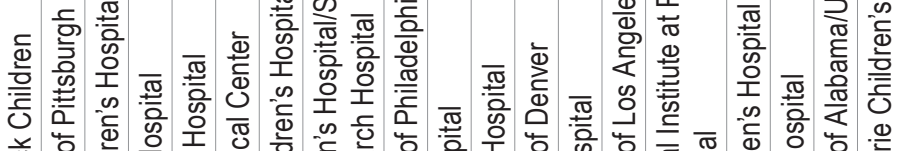

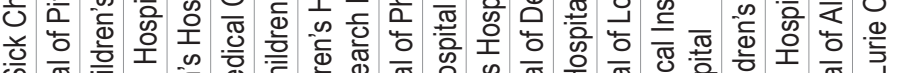

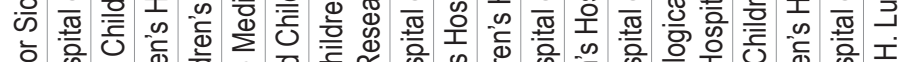

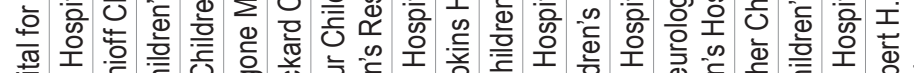

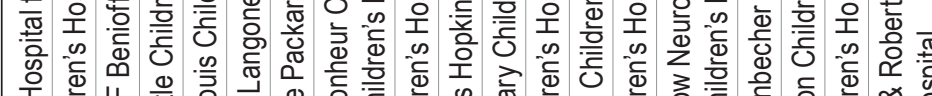

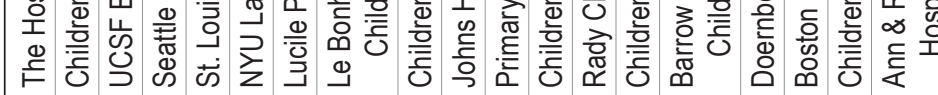

$\sigma \mp \oplus \mp \infty$

กำ

ข

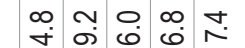

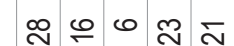

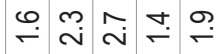

กำ ล

๓

○.

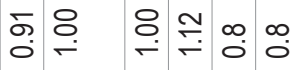

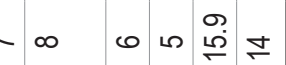

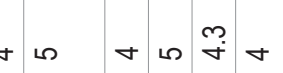

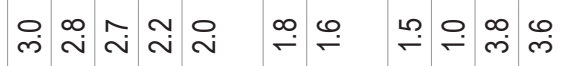

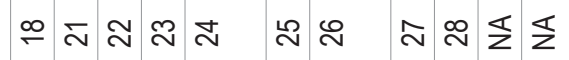

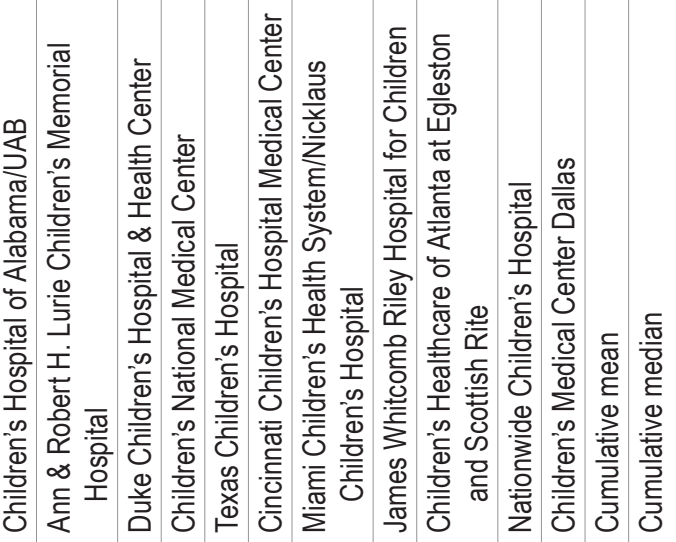

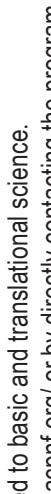

는

造 응

î

咅 들 틍

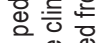

产 产

䒕 뜐

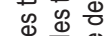

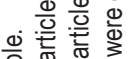
ॠ

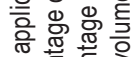
등 Ð "Iㅎำ 
TABLE 6. Dependence of citation impact on academic rank and degree

\begin{tabular}{|c|c|c|c|c|c|c|}
\hline \multirow[b]{2}{*}{ Characteristic } & \multicolumn{2}{|c|}{ Citations per Publication } & \multirow[b]{2}{*}{ p Value } & \multirow[b]{2}{*}{ Publications } & \multirow[b]{2}{*}{ Publications per Author } & \multirow[b]{2}{*}{ Citations } \\
\hline & Mean & Median & & & & \\
\hline Degree & & & $<0.0001$ & & & \\
\hline $\mathrm{MD} / \mathrm{PhD}(\mathrm{n}=21 ; 17 \%)$ & 26.7 & 10.0 & & 811 & 38.6 & 21,690 \\
\hline MD or DO $(n=101 ; 83 \%)$ & 14.0 & 6.0 & & 2103 & 20.8 & 29,503 \\
\hline \multicolumn{7}{|l|}{ Academic rank } \\
\hline Professor $(n=47 ; 39 \%)$ & 21.7 & 8.0 & $<0.0001^{*}$ & 1688 & 35.9 & 36,551 \\
\hline Associate professor $(n=34 ; 29 \%)$ & 13.6 & 6.0 & $0.0007 \dagger$ & 884 & 26.0 & 11,992 \\
\hline Assistant professor $(n=38 ; 32 \%)$ & 8.5 & 4.0 & $<0.0001 \ddagger$ & 370 & 9.7 & 3132 \\
\hline
\end{tabular}

grams. Although two-thirds of the articles in this analysis were pediatric-neurosurgery-specific, adult and mixed adult and pediatric studies averaged significantly more citations per publication (Table 4). This is likely due to the much larger readership and applicability of adult medicine compared with pediatric medicine, especially within neurosurgery and the neurosciences. This finding also shows that the average pediatric neurosurgeon does not exclusively publish papers on pediatric topics.

While about half of the articles in this analysis had a pediatric neurosurgeon as the first or last author, those articles in which the pediatric neurosurgeon was a middle author had significantly greater citation impact. Perhaps this finding is attributable to scenarios in which pediatric neurosurgeons contribute to non-neurosurgery-specific projects that have an overall greater impact, such as clinical neuro-oncology or basic science projects on tumor biology. This also represents the notable frequency of collaboration among pediatric neurosurgeons, either in place of-or in addition to-serving as a principal investigator. Collaborative research, in fact, has been consistently shown to lead to higher citations than if one publishes alone. . $^{20,21}$

As was expected, basic science and translational research papers had significantly higher mean and median numbers of citations per publication-in this case 3 times higher. Whether this ratio of citation impact is proportional to the increased time and resources required for the typical basic or translational project compared with a clinical project is unclear.

When looking just at clinical research, tier 3 articles, which represent in general the lowest quality and least complex research, averaged about half as many citations per publication as tier 1 and 2 articles. This is also expected because research of higher quality and greater complexity (i.e., in terms of the time and resources needed) is more likely to be cited in the future. There was no difference between tier 1 and tier 2, however, which was somewhat unexpected. One contributing factor to this finding is that meta-analyses were classified as tier 2 because they are not original research. Meta-analyses, though, represent high-quality evidence and are likely to be cited frequently. Furthermore, there may be a "ceiling" to citation counts in the very small field of pediatric neurosurgery, mean- ing that even the highest-quality studies may not garner proportionally more citations as they would in other larger fields.

The strong plurality of publications in this analysis was focused on tumor, which accounted for $35 \%$ of all publications and $51 \%$ of all citations. Over $70 \%$ of all basic and translational science articles were tumor focused. Part of this finding may be due to a disproportionate amount of contemporary funding going to neuro-oncology. However, it can also likely be attributed to the fact that this is a subject that crosses over to nonneurosurgery clinicians and scientists. The same applies to spine, epilepsy, and trauma, whereas those subjects that are more neurosurgery-specific-namely Chiari malformation, craniosynostosis, and hydrocephalus-had relatively lower citation impacts.

When looking at tumor, functional/epilepsy, and vascular papers, basic science and translational publications did have a significantly greater number of citations per publication than clinical articles on the same subject. There was no difference in this comparison for spine, trauma, Chiari malformation, or craniosynostosis. However, it is difficult to reliably compare citation impact between clinical versus basic and translational science papers within these latter subjects because only a small number of basic and translational articles were included on each subject.

\section{Author Characteristics}

Faculty members with dual degrees (MD and PhD) were significantly more productive than their MD- or DOonly counterparts. This may be due to some combination of more research training among $\mathrm{PhD}$-trained neurosurgeons and a greater interest in research. It also may be due to $\mathrm{MD} / \mathrm{PhD}$ faculty members publishing more basic science or translational studies, which we have shown to garner more citations per publication, or publishing more papers on topics that have a great citation impact like neuro-oncology.

Consistent with previous reports in both general and pediatric neurosurgery, greater publication productivity is positively correlated with more advanced academic rank..$^{9,11}$ However, the causality in this relationship remains unclear. Rank may progress secondary to reaching a productivity threshold. Alternatively, increased rank may 
lead to more collaborative opportunities (i.e., research networks) or gift authorships as highlighted previously. Likely there is an element of both.

\section{Rankings}

This is the first ranking of pediatric neurosurgery programs that used a contemporary measure of productivity rather than summed career metrics as we used in our prior report. As we have more recently posited, it is much more informative to examine publication productivity through a contemporary lens rather than looking at summed metrics over a faculty member's or institution's lifetime. Using a contemporary discrete 5-year period may highlight recent productivity in historically less-productive programs or stagnation in programs with strong historical reputations. Contemporary metrics and descriptive analyses, as we have done, are far more informative than simply the total number of publications. This goes for all stakeholders, including administrators, department leadership, funding bodies, and program applicants.

In this report, we provide 4 rankings for the 28 programs. Our primary ranking is the $\mathrm{i} h(5)$-index corrected for the number of faculty members [i $h(5)$-index/(number of faculty members)] (Table 5). Correcting for the number of authors is important in the setting of pediatrics where an increase in 1 faculty member can change the program size by $25 \%-33 \%$. Compared with our previous ranking of pediatric fellowship programs by using the cumulative $h$-index, only 1 program (The Hospital for Sick Children) remained in the top 5 and only 4 remained in the top 10 (Seattle Children's Hospital, St. Louis Children's Hospital, and Children's Hospital of Pittsburgh).

We also presented rankings based on our novel metric, the ir(5)-index, which is both corrected and uncorrected for the number of faculty members. The faculty-corrected ir(5)-index was used as the secondary ranking metric. There were only 3 fellowship programs within the top 10

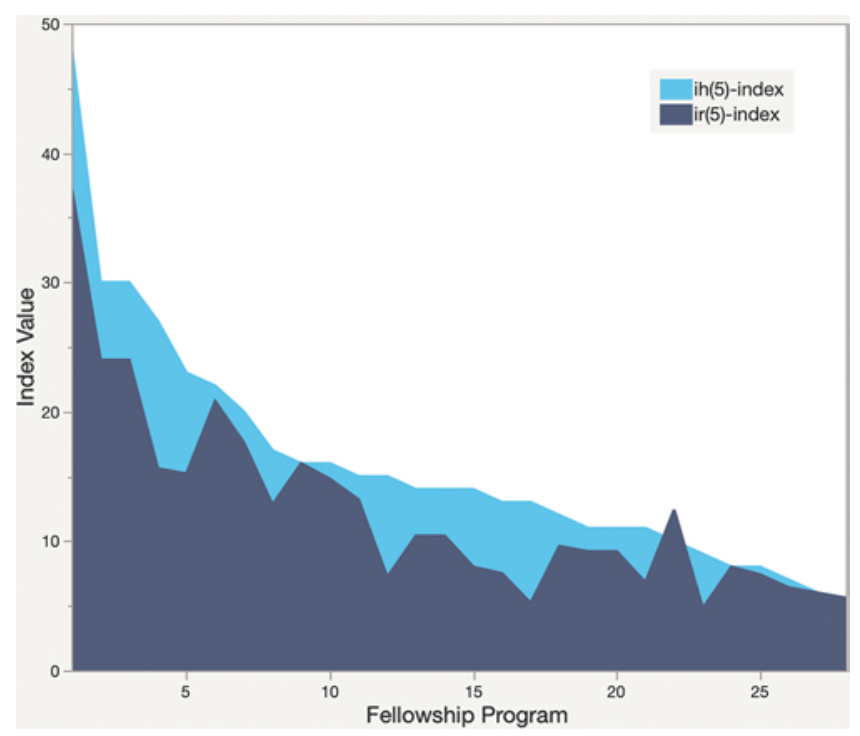

FIG. 1. Comparison of the ih(5)-index and ir(5)-index. The x-axis corresponds to the program rankings by the uncorrected ih(5)-index. Figure is available in color online only. faculty-corrected $\mathrm{i} h(5)$-index list that were not present in the top 10 corrected ir(5)-index list: Lucile Packard Children's Hospital, Children's Hospital of Pennsylvania, and Johns Hopkins were replaced with Primary Children's Hospital, Rady Children's, and Children's Hospital of Los Angeles. This finding implies that, in general, uppertier programs are highly productive both as collaborators and primary investigators. We feel that the ratio of these 2 faculty-adjusted metrics-ir(5)-index to i $h(5)$-index-is an important measure going forward. As shown in Fig. 1 , there is considerable variability in the ratio of these 2 metrics, and on average the authorship-weighted index is lower than the nonweighted index.

Moving forward, more emphasis should be placed on the subject of publication authorship value, particularly because the number of authors has increased significantly in neurosurgical publications, including gift authorship..$^{13}$ Middle authorship represents an unknown when it comes to assessing productivity. There is no question that middle authors invest less time and intellectual effort into a project than the primary, secondary, and senior authors, but not much more can be said beyond that. There is also often significant variability in the contributions of middle authors. For many projects, some middle authors have contributed significantly whereas others contribute minimally or are given authorship as a "gift."

In general, we intentionally did not overemphasize any implications of ranking and we provided multiple methods of ranking to give readers information that can be interpreted and used as appropriate per the situation. Bibliometric indices are only a proxy of departmental productivity, and each has inherent biases and flaws. Furthermore, publication productivity is only 1 of many critical factors that contributes to the strength of an academic training environment. However, given the noticeably increased attention to quantifying and comparing program productivity, we have attempted to incrementally improve the process and provide the most advanced analysis yet produced.

There was no specific face validation performed on these rankings because there is no comparison metric that would serve as a gold standard. The well-publicized US News and World Report rankings incorporate a wide variety of factors and include neurology departments, thereby making comparison with our rankings more "apples-tooranges" than "apples-to-apples."11 Ranking programs by National Institutes of Health funding would provide a more compatible comparison because both focus on research and our previous work would suggest a positive correlation. ${ }^{30}$

\section{Limitations}

There were 2060 unique articles counted with at least 1 author from a pediatric neurosurgery fellowship program included in this analysis. This amounts to roughly 400 articles per year. However, this does not include all articles in the pediatric neurosurgery literature or even all articles published by pediatric neurosurgeons. This publication selection bias is a limitation of the article characteristics analysis because the collected data are restricted to those faculty members working at these 28 institutions. There are many more pediatric neurosurgeons working at other academic centers whose work was not included in this 
study. There are also pediatric neurosurgeons in nontraditional academic settings who were not included, which adds additional bias to these results. We used a 3-tier classification system to acknowledge that publications are not equal in terms of effort and complexity; nevertheless, we accept some criticism of this system.

\section{Conclusions}

This iteration further advances the analysis of publication productivity in pediatric neurosurgery to date. We provide an updated methodology that more appropriately weighs authorship value to accurately quantify and compare contemporary productivity among programs. We used both previously validated metrics and a proposed new metric. We also provide a novel analysis of article and author characteristics that correlate with citation impact. Regular assessment of academic productivity with continual methodological refinement is important to our small field.

\section{Acknowledgments}

We thank Andrew J. Gienapp (Medical Education, Methodist University Hospital and Department of Neurosurgery, University of Tennessee Health Science Center, Memphis, Tennessee) for technical and copyediting, preparation of the manuscript and figures for publishing, and publication assistance with this manuscript.

\section{References}

1. Aoun SG, Bendok BR, Rahme RJ, Dacey RG Jr, Batjer HH: Standardizing the evaluation of scientific and academic performance in neurosurgery-critical review of the " $h$ " index and its variants. World Neurosurg 80:e85-e90, 2013

2. Azer SA, Holen A, Wilson I, Skokauskas N: Impact factor of medical education journals and recently developed indices: can any of them support academic promotion criteria? $\mathbf{J}$ Postgrad Med 62:32-39, 2016

3. Bornmann L, Mutz R, Neuhaus C, Daniel H: Citation counts for research evaluation: standards of good practice for analyzing bibliometric data and presenting and interpreting results. Ethics Sci Environ Polit 8:93-102, 2008

4. Choudhri AF, Siddiqui A, Khan NR, Cohen HL: Understanding bibliometric parameters and analysis. Radiographics 35:736-746, 2015

5. Didegah F, Thelwall M: Which factors help authors produce the highest impact research? Collaboration, journal and document properties. J Informetrics 7:861-873, 2013

6. Hirsch JE: An index to quantify an individual's scientific research output. Proc Natl Acad Sci U S A 102:16569-16572, 2005

7. Jamjoom AA, Wiggins AN, Loan JJ, Emelifeoneu J, Fouyas IP, Brennan PM: Academic productivity of neurosurgeons working in the United Kingdom: insights from the h-index and its variants. World Neurosurg 86:287-293, 2016

8. Jenkins NR: Variation in the h-index and its use in the assessment of academic output. World Neurosurg 87:619-620, 2016

9. Kalra RR, Kestle JR: An assessment of academic productivity in pediatric neurosurgery. J Neurosurg Pediatr 12:262265,2013

10. Kashkoush A, Prabhu AV, Tonetti D, Agarwal N: The neurosurgery match: a bibliometric analysis of 206 first-year residents. World Neurosurg 105:341-347, 2017

11. Khan N, Thompson CJ, Choudhri AF, Boop FA, Klimo P Jr: Part I: The application of the h-index to groups of individuals and departments in academic neurosurgery. World Neurosurg 80:759-765, 765.e1-765.e3, 2013
12. Khan NR, Thompson CJ, Taylor DR, Venable GT, Wham RM, Michael LM II, et al: An analysis of publication productivity for 1225 academic neurosurgeons and 99 departments in the United States. J Neurosurg 120:746-755, 2014

13. King JT Jr: How many neurosurgeons does it take to write a research article? Authorship proliferation in neurosurgical research. Neurosurgery 47:435-440, 2000

14. Klimo P Jr, Michael LM II, Venable GT, Taylor DR: Bibliometrics. J Neurosurg 124:877-878, 2016 (Letter)

15. Klimo P Jr, Venable GT, Khan NR, Taylor DR, Shepherd BA, Thompson CJ, et al: Bibliometric evaluation of pediatric neurosurgery in North America. J Neurosurg Pediatr 14:695-703, 2014

16. Knight J: Academic impact rankings of neurosurgical units in the U.K. and Ireland, as assessed with the h-index. Br J Neurosurg 29:637-643, 2015

17. Lee RP, Venable GT, Roberts ML, Parikh KA, Taylor DR, Khan NR, et al: Five-year institutional bibliometric profiles for 119 North American neurosurgical residency programs: an update. World Neurosurg 95:565-575, 2016

18. Lozano CS, Tam J, Kulkarni AV, Lozano AM: The academic productivity and impact of the University of Toronto Neurosurgery Program as assessed by manuscripts published and their number of citations. J Neurosurg 123:561-570, 2015

19. Madhugiri VS, Sasidharan GM, Subeikshanan V, Dutt A, Ambekar S, Strom SF: An analysis of the citation climate in neurosurgical literature and description of an interfield citation metric. Neurosurgery 76:505-513, 2015

20. Mosbah-Natanson S, Gingras Y: The globalization of social sciences? Evidence from a quantitative analysis of 30 years of production, collaboration and citations in the social sciences (1980-2009). Curr Sociol 62:626-646, 2014

21. Persson O, Glänzel W, Danell R: Inflationary bibliometric values: The role of scientific collaboration and the need for relative indicators in evaluative studies. Scientometrics 60:421-432, 2004

22. Ponce FA, Lozano AM: Academic impact and rankings of American and Canadian neurosurgical departments as assessed using the h index. J Neurosurg 113:447-457, 2010

23. Romanovsky AA: Revised h index for biomedical research. Cell Cycle 11:4118-4121, 2012

24. Rubio DM, Schoenbaum EE, Lee LS, Schteingart DE, Marantz PR, Anderson KE, et al: Defining translational research: implications for training. Acad Med 85:470-475, 2010

25. Sarkiss CA, Riley KJ, Hernandez CM, Oermann EK, Ladner TR, Bederson JB, et al: Academic productivity of US neurosurgery residents as measured by h-index: program ranking with correlation to faculty productivity. Neurosurgery 80:975-984, 2017

26. Schoenfeld AJ, Bhalla A, George J, Harris MB, Bono CM: Academic productivity and contributions to the literature among spine surgery fellowship faculty. Spine J 15:21262131, 2015

27. Taylor DR, Venable GT, Jones GM, Lepard JR, Roberts ML, Saleh N, et al: Five-year institutional bibliometric profiles for 103 US neurosurgical residency programs. J Neurosurg 123:547-560, 2015

28. Thompson DF, Walker CK: A descriptive and historical review of bibliometrics with applications to medical sciences. Pharmacotherapy 35:551-559, 2015

29. Tomei KL, Nahass MM, Husain Q, Agarwal N, Patel SK, Svider PF, et al: A gender-based comparison of academic rank and scholarly productivity in academic neurological surgery. J Clin Neurosci 21:1102-1105, 2014

30. Venable GT, Khan NR, Taylor DR, Thompson CJ, Michael LM, Klimo P Jr: A correlation between National Institutes of Health funding and bibliometrics in neurosurgery. World Neurosurg 81:468-472, 2014 
31. Venable GT, Shepherd BA, Loftis CM, McClatchy SG, Roberts ML, Fillinger ME, et al: Bradford's law: identification of the core journals for neurosurgery and its subspecialties. J Neurosurg 124:569-579, 2016

32. Venable GT, Shepherd BA, Roberts ML, Taylor DR, Khan NR, Klimo P Jr: An application of Bradford's law: identification of the core journals of pediatric neurosurgery and a regional comparison of citation density. Childs Nerv Syst 30:1717-1727, 2014

33. Wilkes FA, Akram H, Hyam JA, Kitchen ND, Hariz MI,

Zrinzo L: Publication productivity of neurosurgeons in Great Britain and Ireland. J Neurosurg 122:948-954, 2015

\section{Disclosures}

The authors report no conflict of interest concerning the materi- als or methods used in this study or the findings specified in this paper.

\section{Author Contributions}

Conception and design: Klimo, Lee, Venable, Khan, Taylor. Acquisition of data: Lee, Xu, Dave, Ajmera, Lillard, Wallace, Broussard, Motiwala, Norrdahl, Howie, Akinduro, Vaughn. Analysis and interpretation of data: Klimo, Lee. Drafting the article: Klimo, Lee. Critically revising the article: Klimo, Lee, Venable. Reviewed submitted version of manuscript: Klimo, Lee, Venable, Vaughn. Approved the final version of the manuscript on behalf of all authors: Klimo. Statistical analysis: Lee. Study supervision: Klimo.

\section{Correspondence}

Paul Klimo Jr: Semmes Murphey, Memphis, TN.pklimo@ semmes-murphey.com. 